\title{
Mechanical structure optimization design of an offshore backfilling plough -fenders
}

\author{
Liquan Wang, Jianguo Qin ${ }^{*}$, .
}

College of Mechanical Electrical Engineering, Harbin Engineering University, Harbin 15000, China

*Corresponding auther. Tel.:15754976820

E-mail address: qjg_ngd@163.com

1.Jianguo Qin, studied at College of Mechanical Electrical Engineering, Harbin Engineering

University, and currently works in Inner Mongol University of Technology.

Keywords: Offshore Backfill; Fender; Mechanical structure; Offshore; uniflow Fluent-structure interaction

\begin{abstract}
Offshore backfilling plough are used for backfilling for laying pipelines in seabed trenches; fenders are the primarily consist of an offshore backfilling plough; Research on the virtual join forces of the fenders is essential for predicting the join forces of the backfilling plough during backfilling. In this work, uniflow Fluid-structure interaction is developed to investigate the Join Forces of fenders under different mechanical structure. To analysis of this resulting data, a new fenders structure was carried out.
\end{abstract}

\section{Introduction}

Offshore pipelines on the seabed laid by a vessel are prone to damage by unexpected artificial and natural disturbances caused by fishing nets, anchors, wave oscillations, and other seabed features and seism. Hence, pipelines are normally

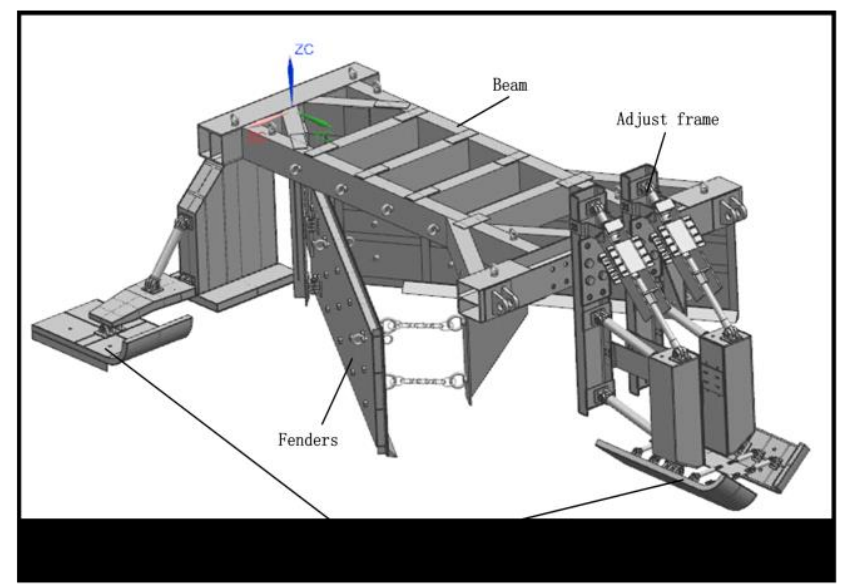

Fig.1. 3-D overview of the offshore backfilling plough buried in trenches using offshore trenchers to prolong their service life and increase the pipelines stability in sea environment. Some of the work was done by backfilling plough.

Mechanical backfilling equipment has been developed based on different pipeline installations and backfill methods in variety of sea and areas and conditions. Consequently, three type of mechanical backfill are classified according to their mode of backfilling as fender mode, fender-reamer mode and jetter mode. Fenders are used both in fender mode and fender-reamer mode.

The offshore backfilling plough consists of four components, as shown in Fig.1. Skid was used to support the system and guide the moving way and adjust the cut depth; Adjust frame was designed to adapt different grooves; Beam to support the overall system. Collect dirt and transport the dirt to grooves was the main work of fenders. When working, the stress of the fenders was the main source of the backfilling plough. Mechanical structure optimization design of the fenders was the most important work to save the system power demand.

An onshore field experimental verification was done, as shown in Fig.2. the model was done in 1:6, through agricultural soil experiment, one of the straight soil, one of

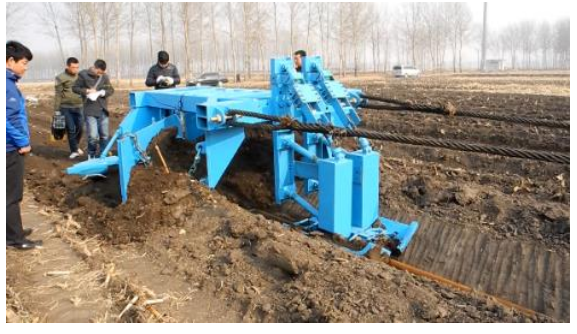

Fig.2. Onshore field experimental 
Table.1. A set of data with the straight fenders results

\begin{tabular}{|c|c|c|c|c|c|c|c|}
\hline \multicolumn{2}{|c|}{ Test project } & \multicolumn{7}{|c|}{} \\
\hline $\begin{array}{c}\text { Angle } \\
\text { between } \\
\text { two } \\
\text { fenders }\end{array}$ & $\begin{array}{c}\text { Speeds } \\
(\mathrm{m} / \mathrm{h})\end{array}$ & \multicolumn{6}{|c|}{ Pull of the straight Fender(KN) } \\
\hline \multirow{2}{6}{$64^{\circ}$} & 200 & 5.12 & 5.63 & 6.10 & 5.69 & 5.58 & 5.49 \\
\cline { 2 - 8 } & 360 & 6.34 & 6.49 & 6.56 & 6.64 & 6.83 & 6.84 \\
\hline
\end{tabular}

the straight fenders results are shown in Table.1. From the results show that the fenders need too much energy to move. The onshore field is that all, What about offshore? Can the mechanical structure of fenders be changed which would save any energy?

\section{Different mechanical structure model of the fenders}

The system was designed to working offshore $500 \mathrm{~m}$, the mechanical structure model was simplified to good in the analysis. Fig.3. is the Simplified model of the fenders for

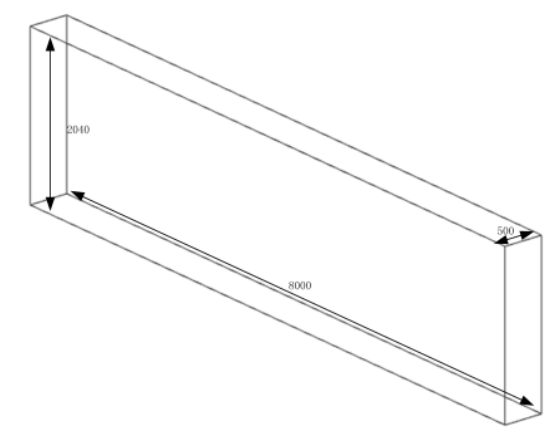

Fig.3. The Simplified model of fenders

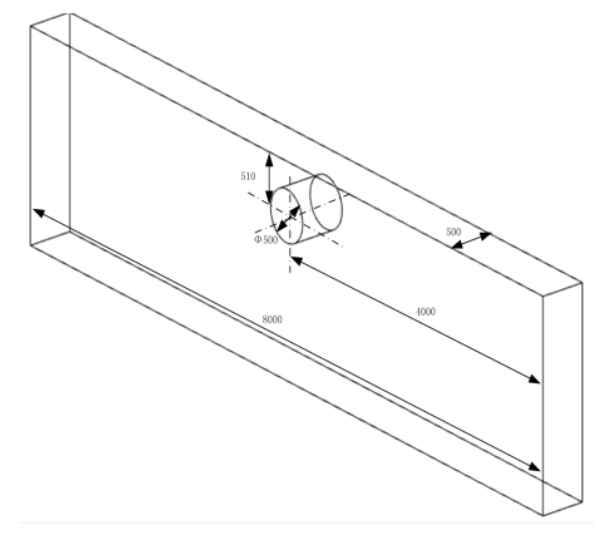

Fig.4. The Simplified improve of

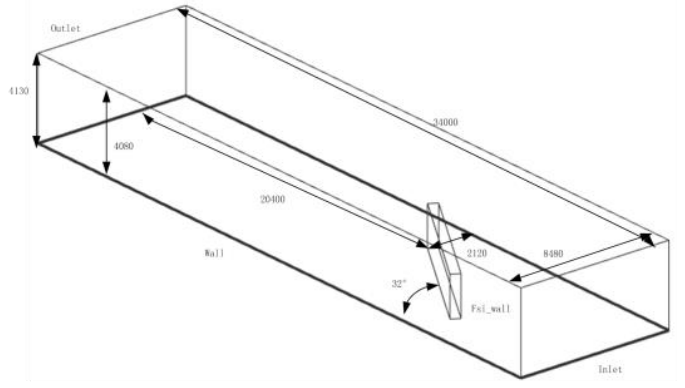

Fig.5. The basic size of analysis module analyze and Fig.4. is the Simplified improve model of the fenders. The analysis model of basic size according to the actual size. Fig.5. is the basic size of analysis model.

\section{Selection of multiphase models}

In this paper, the system was designed to working offshore. When the system work, the fenders push soil and water movement. So, there are two phases. In the simulation, for the sake of convenience, assume the fender is not moving; the water and mud rush to the fenders, and the mud would get to different fender heights; and all phases are incompressible; This is consistent with the Eulerian Model.

\subsection{Volume fraction equation}

The description of multiphase flow as interpenetrating continua incorporates the concept of phasic volume fractions. Volume fractions represent the space occupied by each phase, and the laws of conservation of mass and momentum are satisfied by each phase individually. The derivation of the conservation equations can be done by ensemble averaging the local instantaneous balance for each of the phases or by using the mixture theory approach.

Denoted $\alpha_{q}$ as volume fractions of the volume of phase, the volume of this phase is

$$
V_{q}=\int_{V} \alpha_{q} d V
$$

Where

$$
\sum_{q=1}^{n} \alpha_{q}=1
$$

The effective density of phase $q$ is

$$
\widehat{\rho}_{q}=\alpha_{q} \rho_{q}
$$

where $\rho_{q}$ is the physical density of phase $q$. 
The volume fraction equation may be solved through explicit time discretization. The explicit formulation is time-dependent and the volume fraction is discretized in the following manner:

$$
\frac{\alpha_{q}^{n+1}-\alpha_{q}^{n}}{\Delta t} \mathrm{~V}+\sum_{f}\left(U_{f}^{n+1} \alpha_{q, f}^{n+1}\right)=0
$$

where

$$
\begin{aligned}
& n+1=\text { infex for new (current) time step } \\
& n=\text { infex for previous time step } \\
& \alpha_{q, f}=\text { face value of the } \mathrm{q}^{\text {th }} \text { volume fraction } \\
& \mathrm{V}=\text { volume of cell } \\
& U_{f}=\text { volume flux through the face, } \\
& \text { based on normal velocity }
\end{aligned}
$$

\subsection{Conservation of Mass}

\subsubsection{Continuity equation}

The continuity equation for phase $q$ is

$$
\frac{\partial}{\partial t}\left(\alpha_{q}\right)+\nabla \cdot\left(\alpha_{q} \rho_{q} \overrightarrow{V_{q}}\right)=\sum_{p=1}^{n}\left(\dot{m}_{p q}-\dot{m}_{q p}\right)+S_{q}
$$

where $\vec{V}_{q}$ is the velocity of phase $q$ and $\dot{m}_{p q}$ characterizes the mass transfer from the $p^{\text {th }}$ to $q^{\text {th }}$ phase, and $\dot{m}_{p q}$ characterizes the mass transfer from phase $q$ to phase $p$, and by the continuity equation, we could found that

$$
\begin{aligned}
& \dot{m}_{p q}=-\dot{m}_{q p} \\
& \dot{m}_{p p}=0
\end{aligned}
$$

\subsubsection{Conservation of Momentum}

The momentum balance for phase $q$ yields

$\frac{\partial}{\partial t}\left(\alpha_{q} \rho_{q} \vec{V}_{q}\right)+\nabla \cdot\left(\alpha_{q} \rho_{q} \vec{V}_{q} \vec{V}_{q}\right)=-\alpha_{q} \nabla p+\nabla \cdot \overline{\bar{\tau}}_{q}+$

$\alpha_{q} \rho_{q} \vec{g}+\sum_{p=1}^{n}\left(\vec{R}_{p q}+\dot{m}_{p q} \vec{V}_{p q}-\dot{m}_{q p} \vec{V}_{q p}\right)+\left(\vec{F}_{q}+\vec{F}_{l i f t, q}+\right.$

$\left.\vec{F}_{w l, q}+\vec{F}_{V m, q}+\vec{F}_{t d, q}\right)$

Where $\overline{\bar{\tau}}_{q}$ is the $q^{\text {th }}$ phase stress-strain tensor.

$$
\overline{\bar{\tau}}_{q}=\alpha_{q} \mu_{q}\left(\nabla \vec{V}_{q}+\nabla \vec{V}_{q}^{T}\right)+\alpha_{q}\left(\lambda_{q}-\frac{2}{3} \mu_{q}\right)
$$

Here $\mu_{q}$ and $\lambda_{q}$ are the shear and bulk viscosity of $q, \vec{F}_{q}$ is an external body force, $\vec{F}_{l i f t, q}$ is lift force (described in lift force), $\vec{F}_{w l, q}$ is a wall lubrication force (described in wall lubrication force), $\vec{F}_{V m, q}$ is a virtual mass force, and $\vec{F}_{t d, q}$ is a turbulent dispersion force (in the case of turbulent flows only). $\vec{R}_{p q}$ is an interaction force between phases, and $p$ is the pressure shared by all phases. $\vec{V}_{p q}$ is the interphase velocity.
Equation (7) must be closed with appropriate expressions for interphase force $\vec{R}_{p q}$. This force depends on the friction, pressure, cohesion, and other effects, and is subject to the conditions that $\vec{R}_{p q}=-\vec{R}_{q p}$ and $\vec{R}_{q q}=0$.

A formulas Interaction term are used to represent as the following form:

$$
\sum_{p=1}^{n} \vec{R}_{p q}=\sum_{p=1}^{n} K_{p q}\left(\vec{V}_{p}-\vec{V}_{q}\right)
$$

Where $K_{p q}\left(=-K_{q p}\right)$ is the interphase momentum exchange coefficient, and $\vec{V}_{p}$ and $\vec{V}_{q}$ are the phase velocities.

\subsection{Interphase Exchange Coefficients}

For fluid-fluid flows, each secondary phase is assumed to form droplets or bubbles. This has an impact on how each of the fluids is assigned to a particular phase. For example, in flows where there are unequal amounts of two fluids, the predominant fluid should be modeled as the primary fluid, since the sparser fluid is more likely to form droplets or bubbles. The exchange coefficient for these types of bubbly, liquid-liquid mixtures can be written in the following general form:

$$
K_{p q}=\frac{\rho_{P} f}{6 \tau_{P}} d_{p A_{i}}
$$

where $A_{i}$ is the interfacial area.

where $\tau_{p}$, the "particulate relaxation time", is defined as

$$
\tau_{p}=\frac{\rho_{p d_{p}^{2}}}{18 \mu_{q}}
$$

where $d_{p}$ is the diameter of the bubbles or droplets of phase $p$.

Where, the drag function, is defined by Schiller and Naumann Model, which is used to represent as

$$
f=\frac{C_{D} R_{e}}{24}
$$

Where

$$
C_{D}=\left\{\begin{array}{cl}
24\left(1+0.15 R_{e}^{0.678}\right) / R_{e} R_{e} \leq 1000 \\
0.44 & R_{e}>1000
\end{array}\right.
$$

and $R_{e}$ is the relative Reynolds number. The relative Reynolds number for the primary phase $q$ and secondary phase $p$ is obtained from

$$
R_{e}=\frac{\rho_{q}\left|\bar{V}_{p}-\bar{V}_{q}\right| d_{p}}{\mu_{q}}
$$

The relative Reynolds number for secondary phase $p$ and $r$ is obtained from 


$$
R_{e}=\frac{\rho_{r p}\left|\bar{V}_{r}-\bar{V}_{p}\right| d_{r p}}{\mu_{r p}}
$$

where $\mu_{r p}=\alpha_{p} \mu_{p}+\alpha_{r} \mu_{r}$ is the mixture viscosity of the phases $p$ and $r$.

\subsection{Transport Equations for the Standard $k-\varepsilon$ Model}

The turbulence kinetic energy, $\mathrm{k}$, and its rate of dissipation, $\varepsilon$, are obtained from the following transport equations:

$\frac{\partial}{\partial t}(\rho k)+\frac{\partial}{\partial x_{i}}\left(\rho k u_{i}\right)=\frac{\partial}{\partial x_{j}}\left[\left(\mu+\frac{\mu_{t}}{\sigma_{k}}\right) \frac{\partial k}{\partial x_{j}}\right]+G_{k}+G_{b}-$

$\rho \varepsilon-Y_{M}+S_{k}$

and

$\frac{\partial}{\partial t}(\rho \varepsilon)+\frac{\partial}{\partial x_{i}}\left(\rho \varepsilon u_{i}\right)=\frac{\partial}{\partial x_{j}}\left[\left(\mu+\frac{\mu_{t}}{\sigma_{\varepsilon}}\right) \frac{\partial \varepsilon}{\partial x_{j}}\right]+C_{1 \varepsilon} \frac{\varepsilon}{k}\left(G_{k}+\right.$

$\left.C_{3 \varepsilon} G_{b}\right)-C_{2 \varepsilon} \rho \frac{\varepsilon^{2}}{k}+S_{\varepsilon}$

In these equations, $G_{k}$ represents the generation of turbulence kinetic energy due to the mean velocity gradients, calculated as described in following:

$$
G_{k}=-\rho \overline{u_{\imath}^{\prime} u_{\jmath}^{\prime}} \frac{\partial u_{j}}{\partial x_{i}}
$$

where, $G_{b}$ is the generation of turbulence kinetic energy due to buoyancy, obtained from:

$$
G_{b}=\beta g_{i} \frac{\mu_{t}}{P r_{t}} \frac{\partial T}{\partial x_{i}}
$$

where $P r_{t}$ is the turbulent Prandtl number for energy and $g_{i}$ is the component of the gravitational vector in the $i^{\text {th }}$ direction. For the standard and realizable $k-\varepsilon$ models, the default of $\mathrm{Pr}_{t}$ is 0.85 .

$Y_{M}$ represents the contribution of the fluctuating dilatation in compressible turbulence to the overall dissipation rate, obtained from:

$$
Y_{M}=2 \rho \varepsilon M_{t}^{2}
$$

where $M_{t}$ is the turbulent Mach number, defined as:

$$
M_{t}=\sqrt{\frac{k}{a^{2}}}
$$

Where $a(\equiv \sqrt{\gamma R T})$ is the speed of sound.

$C_{1 \varepsilon}, C_{2 \varepsilon}, C_{3 \varepsilon}$, are constants. $\sigma_{k}$ and $\sigma_{\varepsilon}$ are the turbulent Prandtl number for $k$ and $\varepsilon$. $S_{k}$ and $S_{\varepsilon}$ were defaulted.

\section{Boundary Conditions}

\subsection{Velocity inlet}

The mass flow rate entering a fluid cell adjacent to a velocity inlet boundary is computed as

$$
\dot{m}=\int \rho \vec{V} \cdot d \vec{A}
$$

\subsection{Pressure outlet}

The flow exiting the boundary is subsonic, the strong Averaging approach was in application, the face pressure value $P_{f}$ for subsonic exit flow is computed using the following expression:

$$
P_{f}=P_{e}+(1-F)\left(P_{C}-P_{C_{-} a v g}\right)
$$

where

$P_{C}=$ interior cell pressure at neighboring exit face, $\mathrm{f}$

$P_{c_{\text {avg }}}=$ averaged interior cell pressure at a boundary

$P_{e}=$ specified exit pressure

$F=$ pressure blending factor;

$F=0$ recovers the fully averaged pressure,

$F=1$ recovers the specified pressure.

\subsection{Wall Motion}

The velocity of the fluid at the wall boundary is set to zero (no moving, no wall velocity), so the boundary condition for the velocity becomes:

$$
U_{W a l l}=0
$$

\section{Equation solution}

\subsection{Conservation of Energy}

The volume fraction of each phase is calculated from a continuity equation:

$\frac{1}{\rho_{r p}}\left(\frac{\partial}{\partial t}\left(\alpha_{q} \rho_{q}\right)+\nabla \cdot\left(\alpha_{q} \rho_{q} \vec{V}_{q}\right)=\sum_{p=1}^{n}\left(\dot{m}_{p q}-\dot{m}_{q p}\right)\right)$

where $\rho_{r p}$ is the phase reference density, or the volume averaged density of the $q^{\text {th }}$ phase in the solution domain.

\subsection{Fluid-fluid Momentum Equations}

The conservation of momentum for a fluid phase $q$ is:

$\frac{\partial}{\partial t}\left(\alpha_{q} \rho_{q} \vec{V}_{q}\right)+\nabla \cdot\left(\alpha_{q} \rho_{q} \vec{V}_{q} \vec{V}_{q}\right)=-\alpha_{q} \nabla p+\nabla \cdot \overline{\bar{\tau}}_{q}+$ $\alpha_{q} \rho_{q} \vec{g}+\sum_{p=1}^{n}\left(K_{p q}\left(\vec{V}_{p}-\vec{V}_{q}\right)+\dot{m}_{p q} \vec{V}_{p q}-\dot{m}_{p q} \vec{V}_{q p}\right)+$ $\left(\vec{F}_{q}+\vec{F}_{l i f t, q}+\vec{F}_{w l, q}+\vec{F}_{v m, q}+\vec{F}_{t d, q}\right)$

Here $\vec{g}$ is the acceleration due to gravity. 


\section{Case analysis}

Primary Phase were water, Secondary Phase were mud. The mud flowed from the bottom and the water flowed from all. Here, for the mud,

Density, $\rho_{1}=1030\left(\mathrm{~kg} / \mathrm{m}^{3}\right)$,

Viscosity, $\mu_{1}=2.678(\mathrm{~kg} / \mathrm{m} \cdot \mathrm{s})$;

to the water,

Density, $\rho_{2}=998.2\left(\mathrm{~kg} / \mathrm{m}^{3}\right)$,

Viscosity, $\mu_{1}=0.001003(\mathrm{~kg} / \mathrm{m} \cdot \mathrm{s})$,

Specific Heat $C_{p}=4182(\mathrm{j} / \mathrm{kg} \cdot \mathrm{k})$,

Thermal Conductivity $\lambda=0.6(\mathrm{w} / \mathrm{m} \cdot \mathrm{k})$

Molecular We $\mathrm{Mr}=18.0152(\mathrm{~kg} / \mathrm{kgmol})$,

Standard State EnthalpyS $=-2.858412 \mathrm{e} 8(\mathrm{j} / \mathrm{kgmol})$,

Standard State Entropy

$S m=69902.21(\mathrm{j} / \mathrm{kgmol} \cdot \mathrm{k})$,

L-J Characteristic Length $s_{-} i=1$ (angstrom),

L-J Energy Parameter $e / k=100(\mathrm{k})$

Latent Heat $h_{f g}=2263073(\mathrm{j} / \mathrm{kg})$

Vaporization Temperature $T_{\text {vap }}=284(k)$,

Boiling Point $T_{b p}=373(k)$,

Volatile Component Fraction $f_{v D}=100(\%)$,

Droplet Surface Tension $\sigma=0.0719404(\mathrm{n} / \mathrm{m})$.

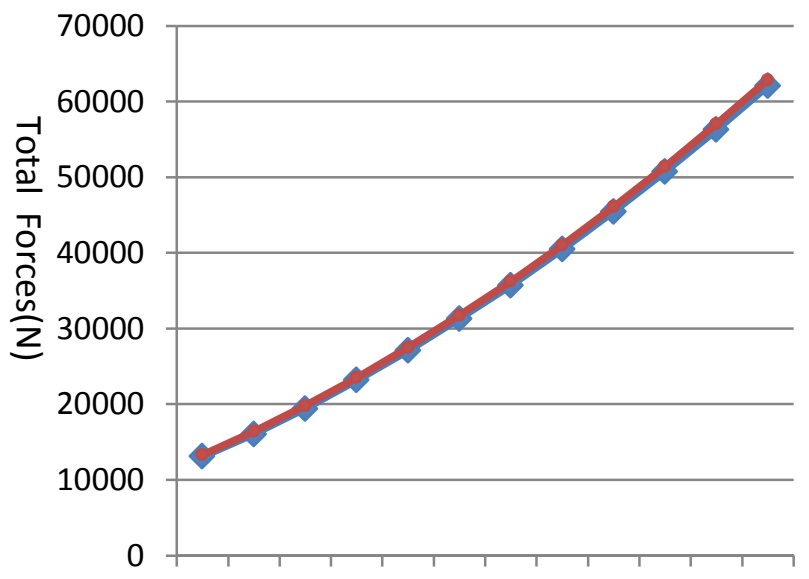

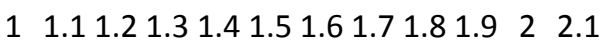

$\operatorname{speed}(\mathrm{m} / \mathrm{s})$

$\sim$ perforated $\quad$ unperforated

Fig 5. Join Forces with the mud reaches $50 \%$ of the fender

and the resulting data. The inlet velocity was from $1 \sim 2.1(\mathrm{~m} / \mathrm{s})$, increasing by 0.1 . The outlet pressure was $5 \mathrm{MP}_{\mathrm{a}}$. The Tables all show that, the unperforated fender is more stressed than the perforated fender, the mud reached the fender $30 \%$ was more obvious than $50 \%$. With the increase of the inlet velocity, the Join Forces of the fender were approximately similar Straight line up. And for the mud to got to the fender $50 \%$, when the velocity was changing from $1.1 \sim 1.4(\mathrm{~m} / \mathrm{s})$, the difference was more obvious; in particular, when the speed was $1.1 \mathrm{~m} / \mathrm{s}$, it reduces the force of $5.3 \%$. Similarly, for the mud reached $30 \%$ of the fender, the overall reduction was close to $1.3 \%$. When the inlet velocity was at the same time, the Join Forces of the fender has changed little with different mud position. These data showed that under the working conditions, the water is relatively large.

Compare table.1. with Fig.5. and Fig.6., the data showed that when the fender was working underwater, the Join Forces of the fender was less than which on land. The main reason is that when working underwater, the fender would be pushed by the water which reduced the Join Forces of the fender.

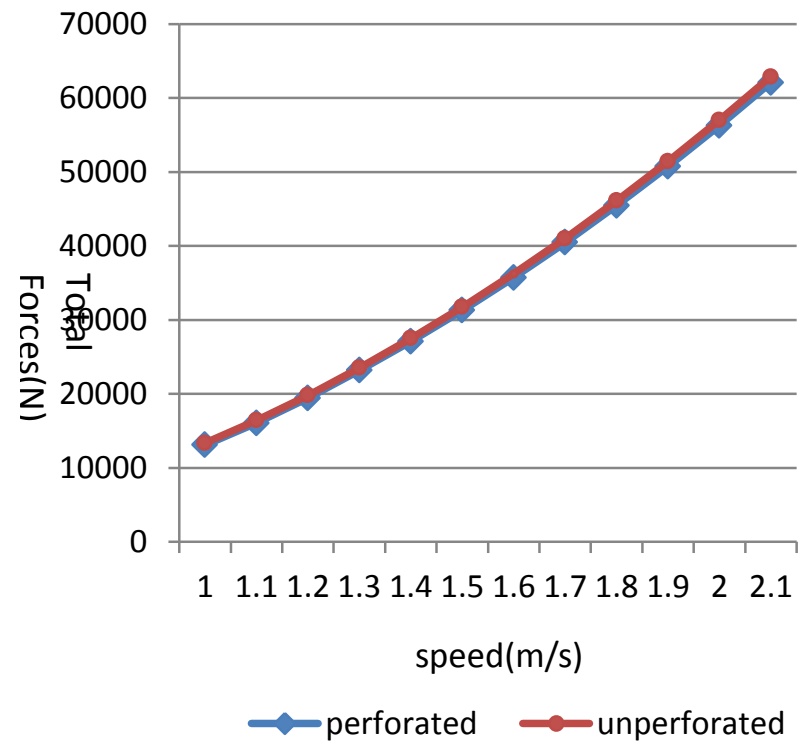

Fig 6. Join Forces with the mud reaches $30 \%$ of the fender

\section{Conclusion}

This paper presents a virtual 3-D uniflow fluid-fluid coupling simulation model of an offshore fender in WORKBENCH. The analysis of the different inlet velocity with different mud position. The analysis of the fender provides the Eulerian Model. Based on this model, several simulation analysis were carried out to predict the fender 
mechanical structure. The conclusion in this paper are follows:

(1) The analysis was consistent with the hypothesis. A perforated fender is less stressed than a unperforated which shows that it is helpful to reduce the Join Forces by increasing the number of holes in the case of the structural strength and the height of the mud.

(2) When the inlet velocity was $2.1(\mathrm{~m} / \mathrm{s})$, the Join Forces of the unperforated fender was $62860 \mathrm{~N}$ where the perforated fender was $62081 \mathrm{~N}$. And when the inlet velocity was $1(\mathrm{~m} / \mathrm{s})$, the Join Forces of the unperforated fender was $13332 \mathrm{~N}$ where the perforated fender was $13088 \mathrm{~N}$. A perforated structure is more efficient than a non-porous structure.

(3) When the mud reaches $50 \%$ of the fender, the inlet velocity was $1.1(\mathrm{~m} / \mathrm{s})$, the Join Forces reduction was more pronounced; consider the mud reaches $30 \%$ of the fender, relatively, a non-porous structure could save more energy with this speed.

\section{References}

[1]Wang L, Gong $\mathrm{H}$, Xing $\mathrm{X}$, et al. Rigid dynamic performance simulation of an offshore pipeline plough[J]. Ocean Engineering, 2015, 94:51-66.

[2]Mikelić, Andro, Guidoboni, et al. Fluid-structure interaction in a pre-stressed tube with thick elastic walls I: the stationary Stokes problem[J]. Networks \& Heterogeneous Media, 2017, 2(3):397-423.

[3]Dunne T, Rannacher R. Adaptive Finite Element Approximation of Fluid-Structure Interaction Based on an Eulerian Variational Formulation[M]// Fluid-Structure Interaction. 2006:110-145.

[4]Valkov B, Rycroft C H, Kamrin K. Eulerian method for fluid-structure interaction and submerged solid-solid contact problems[J]. Eprint Arxiv, 2015, 82(4).

[5]Sugiyama K, Ii S, Shimizu K, et al. A Full Eulerian Method for Fluid-structure Interaction Problems [J]. Procedia Iutam, 2017, 20:159-166.

[6]Abas A, Abdul-Rahman R. Finite Element Analysis of Fluid Structure Interaction in Fully Eulerian Coordinates[J]. Indian Journal of Science \& Technology, 2015.

[7]Kulak R F. A Finite Element Formulation for Fluid-Structure Interaction in Three-Dimensional Space[J].
Journal of Pressure Vessel Technology, 1979, 103(2):183-190.

[8]Majidi S, Afshari A. A ghost fluid method for sharp interface simulations of compressible multiphase flows[J]. Journal of Mechanical Science and Technology, 2016, 30(4):1581-1593.

[9]Karray S, Driss Z, Kchaou H, et al. Numerical simulation of fluid-structure interaction in a stirred vessel equipped with an anchor impeller[J]. Journal of Mechanical Science and Technology, 2011, 25(7):1749-1760.

[10]Sandberg G, Wernberg P A, Davidsson P. Fundamentals of Fluid-Structure Interaction[M]// Computational Aspects of Structural Acoustics and Vibration. Springer Vienna, 2008:23-101.

[11]Eloranta H, Pärssinen T, Saarenrinne P. Fluid-structure interaction of a splitter plate in a convergent channel[J]. Experiments in Fluids, 2005, 39(5):841-855.

[12]Lu L, Su H, Liang Y, et al. Research on Static Stiffness of Hydrostatic Bearing using Fluid-Structure Interaction Analysis[J]. Procedia Engineering, 2012, 29(4):1304-1308.

[13]Löhner R, Cebral J, Yang C, et al. Large-scale fluid-structure interaction simulations $[\mathrm{J}]$. Computing in Science \& Engineering, 2004, 6(3):27-37.

[14]Tang Z J, Shen J L, Liu P L, et al. The simulation of paddle based on workbench[C]// Iet International Conference on Information Science and Control Engineering. IET, 2012:3.28-3.28. 